\title{
Femoral bone defect healing using two novel biocompatible degradable materials
}

\author{
Robert Srnec ${ }^{1}$, Andrea Nečasová ${ }^{1}$, Pavel Proks ${ }^{2}$, Miša Škorič ${ }^{3}$, Zita Filipejová ${ }^{4}$ \\ Lucy Vojtová ${ }^{5}$, Alois Nečas ${ }^{1,6}$ \\ ${ }^{1}$ University of Veterinary and Pharmaceutical Sciences Brno, Faculty of Veterinary Medicine, \\ Small Animal Clinic, Department of Surgery and Orthopaedics, Brno, Czech Republic \\ ${ }^{2}$ University of Veterinary and Pharmaceutical Sciences Brno, Faculty of Veterinary Medicine, \\ Small Animal Clinic, Department of Diagnostic Imaging, Brno, Czech Republic \\ ${ }^{3}$ University of Veterinary and Pharmaceutical Sciences Brno, Faculty of Veterinary Medicine, \\ Department of Pathological Morphology and Parasitology, Brno, Czech Republic \\ ${ }^{4}$ University of Veterinary and Pharmaceutical Sciences Brno, Faculty of Veterinary Medicine, \\ Small Animal Clinic, Department of Medicine, Brno, Czech Republic \\ ${ }^{5}$ Brno University of Technology, CEITEC - Central European Institute of Technology, \\ Advanced Biomaterials Group, Brno, Czech Republic \\ ${ }^{6}$ General University Hospital in Prague, Praha, Czech Republic
}

\author{
Received November 26, 2019 \\ Accepted May 26, 2020
}

\begin{abstract}
This study was conducted as an in vivo experiment in adult miniature pigs with the aim to test two new biomaterials. An iatrogenic defect was made into the central femoral diaphysis in the experimental animals and subsequently fixated by bridging plate osteosynthesis. Into the defect we implanted a cancellous autograft (control group), a pasty injectable scaffold (EXP A), and a porous $3 \mathrm{D}$ cylinder (EXP B). Radiological examination was performed in all animals at 0,10 , 20,30 weeks after surgical procedure and histological assessment was performed. In the newly formed bone the osteoblastic activity was monitored. In terms of radiology, the most effective method was observed in the control group (completely healed 100\%) compared to experimental groups EXP A (70.0\%) and EXP B (62.5\%). Histological assessment showed a higher cell count in the place of bone defect in the control group compared to experimental groups. Between the experimental groups, a higher count of bone marrow cells was found in group EXP B. Both newly developed biomaterials seem to be suitable as replacements for large bone defects, having good workability and applicability. However, compared to the control group treated with a cancellous autograft, the newly formed bone did not reach the same number of cells settling in and in some cases, full radiological healing was not reached. Nevertheless, the material was found to be grown into the original bone in all cases within the experimental groups. The new biomaterials have a great potential as a substitute in the treatment of large bone defects.
\end{abstract}

Pig, biomaterial, scaffold, animal model

A large part of scientific research in the field of development of new biomaterials is dedicated to the healing of bone defects. This topic is especially important due to the constant need to find bone tissue replacement in cases of major bone tissue loss in patients. In cases of comminutive fractures with bone tissue loss, corrective osteotomies, replacements of neoplastic tissues and revisional osteosynthesis, it is necessary to obtain suitable material to fill up the bone defect.

As a golden standard, in the majority of cases autologous cancellous, cortical or corticalcancellous grafts are currently used in clinical medicine. Although there are great biological advantages associated with these grafts, their major drawbacks include insufficient availability of the materials, increased invasiveness and length of the surgical procedure.

Even though there are many commercially produced materials available, the current progress requires the development of new materials not only in terms of applicability,

Address for correspondence:

MVDr. Robert Srnec, Ph.D.

Department of Surgery and Orthopaedics

Small Animal Clinic

Faculty of Veterinary Medicine

University of Veterinary and Pharmaceutical Sciences Brno

Palackeho tr. 1946/1, 61242 Brno, Czech Republic

Phone: +420 $541562342 ;+420775385533$

E-mail: srnecr@vfu.cz

http://actavet.vfu.cz/ 
but also in relation to the biological support for the original tissue to heal faster and more effectively.

General requirements for all biomaterials suitable for implantation include biocompatibility, biodegradability, mechanical properties, scaffold architecture and manufacturing technology (O'Brien 2011). The diamond concept of bone fracture healing interactions includes the utilisation of growth factors, osteoconductive scaffolds, osteogenic cells and optimal mechanical environment (Giannoudis et al. 2008).

Our aim was to develop a biomaterial with good biological and application properties, whereas the mechanical stability was left upon the internal fracture fixation. The goal was to obtain an easily applicable material with the possibility of good shape adapting to the conditions of the bone tissue defect and a potential of good cell migration within the material.

The secondary goal for for the new material was its ability to be used as a carrier of bioactive substances or drugs.

\section{Materials and Methods}

The study was designed as an in vivo experiment in adult miniature pigs of the Minnesota breed. The observed group of experimental animals included both females and castrated males of an average age of 2-4 years and an average weight of $70.2 \pm 18.3 \mathrm{~kg}$.

During the experiment, the animals were put under general anaesthesia. Lateral approach to the diaphysis of the left femur was performed, and an iatrogenic defect was made in the central part of the diaphysis identically in all individuals. A $10 \mathrm{~mm}$ long defect was created by cutting out a whole piece of the bone using an oscillating saw. Subsequently, internal fixation of the iatrogenic defect was done by osteosynthesis with the use of locking implants. LCP implants (LCP steel plate 4.5/5.0 broad, 7 holes, $134 \mathrm{~mm}$ length, DePuy Synthes, Johnson and Johnson, USA) and locking screws (steel locking screw ø $5.0 \mathrm{~mm}$, self-tapping, DePuy Synthes, Johnson and Johnson, USA) were used for the fixation. Three locking screws with biocortical introduction were used equally in both proximal and distal fragments.

Experimental animals were divided into 3 groups. In the control group $\left(\mathrm{n}_{\mathrm{C}}=8\right)$ the defect in the central part of diaphysis was filled in with a cancellous autograft collected from the left proximal humeral metaphysis.

In the first experimental group $\left(\mathrm{n}_{\mathrm{A}}=10\right)$ the defect was filled in with a newly synthetised biomaterial of pasty injectable consistency (Plate I, Fig. 1). This material was prepared from $2 \mathrm{~g}$ of powder mixture mixed together with $3 \mathrm{ml}$ of autologous heparinised blood shortly before implantation during the surgical intervention.

One dose of $2 \mathrm{~g}$ of powder mixture contained $0.6 \mathrm{~g}$ of bovine freeze-dried collagen (bovine lyophilized collagen $100 \%$, Collado, s.r.o., Brno, Czech Republic) and $1.4 \mathrm{~g}$ of resorbable bioceramics with a mean particle size of $4.21 \mu \mathrm{m}$. The bioceramics contained $89 \mathrm{wt} \%$ of calcium phosphate and $11 \mathrm{wt} \%$ of calcium pyrophosphate (Fluka, Switzerland).

In the second experimental group $\left(n_{B}=8\right)$, a 3D porous collagenous cylinder was freely inserted between the ends of the main bone fragments using the "press and fit" method (Plate I, Fig. 2). The highly porous collagen/ bioceramics composite cylindrical foams were fabricated by the freeze-drying method according to Prosecká et al. (2015). Briefly, $1 \mathrm{wt} \%$ concentration of aqueous collagen suspension (bovine lyophilized collagen $100 \%$ - Collado, s.r.o., Brno, Czech Republic) and 1\% wt. of bioceramics with a mean particle size of $4.21 \mu \mathrm{m}$ (the bioceramics contained $89 \mathrm{wt} . \%$ of calcium phosphate and $11 \mathrm{wt} . \%$ of calcium pyrophosphate - Fluka, Switzerland) were mixed together using a disintegrator (5 $000 \mathrm{rpm}$ for $5 \mathrm{~min}$ ). Six millilitres of collagen/bioceramic suspension were poured into each hole of a 12-hole well-plate with the addition of $4 \mathrm{mg}$ of nanofibres (polycaprolactone nanofibres, $45000 \mathrm{Da}$, Sigma-Aldrich, MO, USA) prepared by the "wet-spinning" method (Míša Rampichová, Matěj Buzgo, Prague, Czech Republic) used in collagen foam. Samples were freeze-dried in Epsilon 2-10D LSCplus lyophilizer (Martin Christ Gefriertrocknungsanlagen $\mathrm{GmbH}$, Osterode am Harz, Germany) for two days (the temperature of the icecondenser and the shelf temperature was $-80{ }^{\circ} \mathrm{C}$ and $-50{ }^{\circ} \mathrm{C}$, respectively). Dried samples were chemically crosslinked via EDC/NHS mixture, purified and freeze-dried again as mentioned above.

Closure of surgical wound, antibiotic coverage, pain management and post operative management were conducted identically in all animals in compatibility with the approved experimental project, according to the welfare and ethical principles of the handling of experimental animals.

All animals were further observed for the time period of 30 weeks after the surgical intervention.

The overall health status of all animals was monitored and radiological evaluation of the bone defect was performed at the intervals of $0,10,20$ and 30 weeks, after surgical procedure. Radiographic images of the left femur in mediolateral and craniocaudal projections were obtained in general anaesthesia and the healing status of the bone was evaluated by the same radiologist classified as healed, partially healed or unhealed.

Also the presence of periosteal reaction (according to quantity grade 1,2 or 3) and the possibility of formation of a new hypertrophic callus (yes/no) were evaluated.

All animals were euthanized lege artis after 30 weeks and segments of bony tissues in the implanted area including the adjacent tissues were dissected and sent for histological examination. 
Histological examination was performed in sections obtained from the observed areas, stained by haematoxylineosin and cellularity/osteoblastic activity in the new formed bone tissue was evaluated (examined in the vision field at a $\times 400$ magnification as an average amount of cell in 10 vision fields).

The obtained data were evaluated by basic descriptive statistical methods. Parametric $t$-test for unpaired comparison (Kyplot version 2.0 beta 15-32 bit) was used for statistical data evaluation.

\section{Results}

In terms of radiological evaluation, the process of bone defect healing was the most effective and had the fastest progress in the control group, where the bone defect was filled in with the autogenous cancellous bone graft, and no significant periosteal reaction or hypertrophic callus formation was observed (Plate II, Fig. 3). In the majority of cases in both experimental groups the bone healing process resulted in a complete healing of the bone defect, while in a minority of cases it resulted in a partial healing of the defect. However, the healing progress of the defect was slower and with a stronger local reaction (periosteal reaction, hypertrophic callus formation) compared to the control group (Tables 1 and 2, Figs 4 and 5 [Plate II]). From the histological point of view, the newly formed compact bone in the control group (defect filled in with cancellous bone autograft) was of a higher density with a higher presence of osteons, in comparison with experimental groups EXP A and EXP B. Furthermore, a significantly higher cell density localized in the newly formed bony structures in the place of defect was noted in the control group in comparison with experimental groups EXP A (paste injectable scaffold with autologous blood, $P \leq 0.01$ ) and EXP B (prefabricated 3D porous collagenous cylinder, $P \leq 0.001$ ).

Table 1. Radiological evaluation of bone healing.

\begin{tabular}{lllll}
\hline Group & & \multicolumn{2}{c}{ Healing status } \\
& & Nonunion & Partial & Complete \\
\hline \multirow{2}{*}{$\begin{array}{l}\text { 10 weeks after } \\
\text { surgical procedure }\end{array}$} & Control $(\mathrm{n}=7)$ & $0(0 \%)$ & $7(100 \%)$ & $0(0 \%)$ \\
& EXP A - paste scaffold $(\mathrm{n}=10)$ & $3(30 \%)$ & $7(70 \%)$ & $0(0 \%)$ \\
20 weeks after & Control $(\mathrm{n}=7)$ & $3(37.5 \%)$ & $5(62.5 \%)$ & $0(0 \%)$ \\
surgical procedure & EXP A - paste scaffold $(\mathrm{n}=10)$ & $0(0 \%)$ & $1(14.3 \%)$ & $6(85.7 \%)$ \\
& EXP B - 3D cylinder scaffold $(\mathrm{n}=8)$ & $3(0 \%)$ & $4(40 \%)$ & $6(60 \%)$ \\
30 weeks after & Control $(\mathrm{n}=7)$ & $0(0 \%)$ & $0(0 \%)$ & $7(100 \%)$ \\
surgical procedure & EXP A - paste scaffold $(\mathrm{n}=10)$ & $0(0 \%)$ & $3(30 \%)$ & $7(70 \%)$ \\
& EXP B - 3D cylinder scaffold $(\mathrm{n}=8)$ & $0(0 \%)$ & $3(37.5 \%)$ & $5(62.5 \%)$ \\
\hline
\end{tabular}

Table 2. Radiological evaluation of the periosteal reaction and hypertrophic callus formation.

\begin{tabular}{|c|c|c|c|c|c|c|c|}
\hline \multirow[t]{2}{*}{ Group } & & \multicolumn{4}{|c|}{ Periosteal reaction } & \multicolumn{2}{|c|}{$\begin{array}{c}\text { Hypertrophic } \\
\text { callus }\end{array}$} \\
\hline & & No & Grade 1 & Grade 2 & Grade 3 & Yes & No \\
\hline \multirow{3}{*}{$\begin{array}{l}10 \text { weeks after } \\
\text { surgical procedure }\end{array}$} & Control $(n=7)$ & 5 & 1 & 1 & 0 & 1 & 6 \\
\hline & EXP A - paste scaffold $(n=10)$ & 1 & 3 & 3 & 3 & 4 & 6 \\
\hline & EXP B - 3D cylinder scaffold $(n=8)$ & 7 & 1 & 0 & 0 & 1 & 7 \\
\hline \multirow{3}{*}{$\begin{array}{l}20 \text { weeks after } \\
\text { surgical procedure }\end{array}$} & Control $(n=7)$ & 5 & 2 & 0 & 0 & 1 & 6 \\
\hline & EXP A - paste scaffold $(n=10)$ & 5 & 2 & 1 & 2 & 4 & 6 \\
\hline & EXP B - 3D cylinder scaffold $(n=8)$ & 8 & 0 & 0 & 0 & 1 & 7 \\
\hline \multirow{3}{*}{$\begin{array}{l}30 \text { weeks after } \\
\text { surgical procedure }\end{array}$} & Control $(n=7)$ & 7 & 0 & 0 & 0 & 1 & 6 \\
\hline & EXP A - paste scaffold $(n=10)$ & 8 & 0 & 0 & 2 & 3 & 7 \\
\hline & EXP B - 3D cylinder scaffold $(n=8)$ & 8 & 0 & 0 & 0 & 1 & 7 \\
\hline
\end{tabular}


When comparing the experimental groups EXP A and EXP B, higher counts of bone marrow cells were present in the newly formed lamellar bone in experimental group B (porous 3D collagenous cylinder). The proliferation of perivascular and endothelial cells of arterial anastomoses with deposition of lamellar bone and osteon formation was higher in experimental group EXP B compared to experimental group EXP A $(P \leq 0.001)$. A mild deposition of trabecules of primary bone activated by periosteal osteoblasts (periosteal reaction at the place of periosteum disruption) was found in the place of defect in the periosteum in all groups. Morphological look of the newly formed bone in the place of defect was similar in all groups (control, EXP A and EXP B).

\section{Discussion}

One of the important characteristics of the bone substitute in cases of spatially challenging defects of bone tissue or dissected bones is the ability to fill in the missing bone tissue. One of the possible options is to prepare the exact shape of the missing tissues based on previous computed tomography scan. These procedures are usually results of very modern technologies such as digital navigation and 3D printing (Inzana et al. 2014; Zhang et al. 2019). In some cases it is not possible to apply this approach in advance (emergency care) or the spatial arrangement is changeable up to the moment of fracture fixation (comminutive fracture). In such cases other types of bone tissue substitutes are convenient to fill in the defect thanks to the plastic attributes of injectable scaffolds. The advantage of these injectable substitutes is the possibility of mini-invasive procedures (Liu et al. 2019). A disadvantage of the pasty injectable substitutes is smaller mechanical support at the localisation of the bone defect. In our case we aimed for two possibilities of application.

The first application type was a pasty injectable substitute (group EXP A), which was made by mixing the powder mixture with autologous blood. This scaffold type showed very good workability and easy applicability into the place of bone defect. In previous studies we developed an injectable substitute for smaller bone defects, which can be useful in cases such as filling in defects originating from a removal of internal fracture fixation. The developed substitute was a hydrogel material based with very good properties but also with a major disadvantage when it came to solubility. After application into the bone defect with a higher degree of bleeding at the site where the substitute was implanted, dissolution and washing out of the injectable scaffold started to occur (Srnec et al. 2018). For the new variant of the injectable substitute for extensive bone defects (EXP A) this drawback was avoided. In contrast, during the contact of the scaffold of pasty consistency with blood the bonds within the scaffold were strengthened and the pasty structure was changed to gellike structure which was resistant to washing out by bleeding. This excellent property was found to be indispensable for the filling of major bone defects. As a possible disadvantage we could consider the lesser porosity of the scaffold, which leads to decreased levels of cell elements within the scaffold. With this type of scaffold we also noticed during radiological examination after 10 and 20 weeks post surgery a high occurrence of periosteal reaction and formation of hypertrophic bone callus. We explain this fact as a sign of an infectious process. The process of obtaining peripheral blood in miniature pigs comes with a high risk of failing to maintain complete asepsis, which could have lead to contamination of the scaffold and signs of a septic process. This process was gradually eliminated in all cases by the defence mechanisms in the body, however, we observed a prolonged time of healing in this experimental group. Macroscopically, the defects healed in all animals in the control and in the experimental groups. During radiological evaluation, we observed signs of bone healing process as soon as 30 weeks after surgical procedure in $30 \%$ of the cases in experimental group A and $37.5 \%$ of the cases in experimental group $\mathrm{B}$, but we were still able to recognize the original osteotomic line. These cases 
were later considered from the radiological point of view as partially healed. Although we presume that in all of these cases it would have led to a full radiological healing of the defect, but in a prolonged time period. We can interpret the results of histological examinations of the samples in a similar way. Histologically, there are no major differences between the newly formed bone tissue in the control group and the experimental groups. A difference was seen in the counts of cells inhabiting the newly formed bone tissue. This could mean that the process of inhabiting the bone tissue with cells is slower, leading to a prolonged time of healing.

As the second substitute type (experimental group EXP B) we used a scaffold that was shaped during its processing (prefabricated 3D cylinder), but the structure of this scaffold was spongy with a very good workability. During surgery, the scaffold can be shaped into the exact form of the bone defect and can be applied by pressing the spongy substitute between bone fragments ("press and fit" method). Such concepts have been used by other researchers (Schaefer et al. 2002). Comparing the newly invented scaffold types, radiological examination yielded similar results. Histological examination showed better cellularity and cell population of the newly formed bone tissue in the case of 3D cylinder (EXP B), which can be attributed to a better porosity of the scaffold in comparison with the pasty injectable form. Nevertheless, this difference had no influence on the clinical or radiological healing of the bone defect when both scaffold types were compared.

Generally, biological material or artificial biomaterial can be used for bone tissue substitutes. Based on the place of origin, biological substitutes can be classified as autograft, allograft or xenogeneic. From a clinical point of view, autograft substitutes are still considered a golden standard, but they increase the invasivity for the patient and do not usually offer sufficient amount of material in cases of major bone loss (O'Brien 2011; Zhang et al. 2019). Due to these reasons we chose the application of an autologous cancellous graft into the iatrogenic defect in femur as a control group for comparison of bone healing after the application of new synthetic materials.

The clinical use of xenogenous materials is described less often with uncertain long-term effect (Hesse et al. 2010; Zhang et al. 2019). Biomaterials seem to be an ideal choice for the future of bone defect healing. Aside from standardized attributes, it is possible to easily use sufficient amounts according to the size of the defect and to enrich these materials by bioactive substances and/or drugs. The most commonly used scaffolds for bone substitutes are materials based on collagen and hydroxyapatite. Collagen, as the most common protein in the body, provides structural stability, and together with hydroxyapatite it is a major component of the bone (O'Brien 2011). Another frequently used natural polymer for development of bone substitute is polysaccharide chitosan (Zhang et al. 2019). Numerous studies have used these basic components for the development of bone tissue substitutes (Du et al. 1999; Liao et al. 2004; Pighinelli and Kucharska 2013; Sathiyavimal et al. 2019; Wahl and Czernuszka 2006; Wang et al. 2019; Zhao et al. 2002). Beta-tricalcium phosphate is now one of the most common synthetic materials used for bone reconstruction in orthopaedic and maxillofacial surgery. This biomaterial is easier to be absorbed than hydroxyapatite and highly biocompatible (Guillaume 2017; Zhang et al. 2019). In a study by Mayr et al. (2015) microporous $\beta$-tricalcium phosphate was used alone for a bone defect in sheep. After 24 weeks, highly advanced resorption of the $\beta$-tricalcium phosphate implant and trabecular structure of the new bone was seen (Mayr et al. 2015).

Due to the reasons mentioned above, as a basic substance for development of new biomaterials we preffer to use collagen combined with $\beta$-tricalcium phosphate in both scaffolds; the time set for bone healing was 30 weeks for both biomaterials. In the case of the porous $3 \mathrm{D}$ cylinder (EXP B), the basic components of the scaffold were enriched by poly $(\varepsilon$-caprolactone) nanofibres. The positive potential of these nanofibres for bone tissue engineering has been published (Yoshimoto et al. 2003). 
In conclusion, both new biomaterials seem to be suitable substitutes for the filling of large bone defects. They show a very good workability and applicability. Compared to the control group treated with an autogenous cancellous graft, the newly formed bone tissue did not reach the same level of cell settlement and in some cases there was not a full radiological healing of the defect.

Although the radiological healing was not found to be complete in all cases, the biomaterial was detected to be grown into the bone in all cases. The new biomaterials therefore have a high potential to be used as a substitute in cases with large bone loss. Aside from the availability of sufficient amounts of these substitutes, another major advantage is the possibility to optimise the healing by supplementation of drugs or bioactive substances.

In this field, we expect further development of the potential of the material and modifications of these bone substitutes. The results of this study show that a strict aseptic approach has to be kept during the implantation process. Possible contamination of the scaffold can be seen and occurs more often in the case of using a synthetic substitute rather than in the case of using an autogenous biological graft during the healing process.

\section{Acknowledgement}

This work was supported by the project "Bones, tendons, and ligaments extent defects healing with using of new biomaterials" by the Ministry of Health of the Czech Republic (Czech Health Research Council MZ-VES project no. 16-28637A).

\section{References}

Du C, Cui FZ, Zhu XD, de Groot K 1999: Three-dimensional nano-HAp/collagen matrix loading with osteogenic cells in organ culture. J Biomed Mater Res 44: 407-415

Giannoudis PV, Einhorn TA, Schmidmaier G, Marsh D 2008: The diamond concept - open questions. Injury, Int J Care Injured 39S2: S5-S8

Guillaume B 2017: Filling bone defects with $\beta$-TCP in maxillofacial surgery: a review. Morphol 101: 113-119

Hesse E, Kluge G, Atfi A, Correa D, Haasper C, Berding G, Shin H, Viering J, Länger F, Vogt PM, Krettek CH, Jagodzinski M 2010: Repair of a segmental long bone defect in human by implantation of a novel multiple disc graft. Bone 46: 1457-1463

Inzana JA, Olvera D, Fuller SM, Kelly JP, Graeve OA, Schwarz EM, Kates SL, Awad HA 2014: 3D printing of composite calcium phosphate and collagen scaffolds for bone regeneration. Biomaterials 35: 4026-4034

Liao SS, Cui FZ, Zhang W, Feng QL 2004: Hierarchically biomimetic bone scaffold materials: nano-HA/collagen/ PLA composite. J Biomed Mater Res Part B Appl Biomater 69B: 158-165

Liu C, Wu J, Gan D, Li Z, Shen J, Tang P, Luo S, Li P, Lu X, Zheng W 2019: The characteristics of mussel-inspired nHA/OSA injectable hydrogel and repaired bone defect in rabbit. J Biomed Mater Res B Appl Biomater 108: 1814-1825

Mayr HO, Suedkamp NP, Hammer T, Hein W, Hube R, Roth PV, Bernstein A 2015: $\beta$-Tricalcium phosphate for bone replacement: Stability and integration in sheep. J Biomech 48: 1023-1031

O’Brien FJ 2011: Biomaterials \& scaffolds for tissue engineering. Mater Today 14: 88-95

Pighinelli L, Kucharska M 2013: Chitosan-hydroxyapatite composites. Carbohydrate Polymer 93: 256-262

Prosecka E, Rampichova M, Litvinec A, Tonar Z, Kralickova M, Vojtova L, Kochova P, Plencner M, Buzgo M, Mickova A, Jancar J, Amler E 2015: Collagen/hydroxyapatite scaffold enriched with polycaprolactone nanofibers, thrombocyte-rich solution and mesenchymal stem cells promotes regeneration in large bone defect in vivo. J Biomed Mater Res Part A 103: 671-682

Sathiyavimal S, Vasantharaj S, LewisOscar F, Pugazhendhi A, Subashkumar R 2019: Biosynthesis and characterization of hydroxyapatite and its composite (hydroxyapatite-gelatin-chitosan-fibrin-bone ash) for bone tissue engineering applications. Int J Biol Macromol 129: 844-852

Schaefer D, Martin I, Jundt G, Seidel J, Heberer M, Grodzinsky A, Bergin I, Vunjak-Novakovic G, Freed LE 2002: Tissue-engineered composites for the repair of large osteochondral defects. Arthritis Rheum 46: 2524-2534

Srnec R, Divin R, Skoric M, Snasil R, Krbec M, Necas A 2018: Use of the peptigel with nanofibres in the bone defects healing. Acta Chir Orthop Traumatol Čechosl 85: 359-365

Wahl DA, Czernuszka JT 2006: Collagen-hydroxyapatite composites for hard tissue repair. Eur Cells Mater 11: 43-56

Wang W, Cao N, Dong J, Boukherroub R, Liu W, Li Y, Cong H 2019: Chitosan/hydroxyapatite modified carbon/carbon composites: synthesis, characterization and in vitro biocompatibility evaluation. RSC Adv 9: 23362-23372 
Yoshimoto H, Shin YM, Terai H, Vacanti JP 2003: A biodegradable nanofibre scaffold by electrospinning and its potential for bone tissue engineering. Biomaterials 24: 2077-2082

Zhang Q, Wu W, Qian CH, Xiao W, Zhu H, Guo J, Meng Z, Zhu J, Ge Z, Cui W 2019: Advanced biomaterials for repairing and reconstruction of mandibular defects. Mat Sci Eng C 103: 109858

Zhao F, Yin Y, Lu WW, Leong Ch, Zhang W, Zhang J, Zhang M, YaoK 2002: Preparation and histological evaluation of biomimetic three-dimensional hydroxyapatite/chitosan-gelatin network composite scaffolds. Biomaterials 23: 3227-3234 
Plate I

Srnec R. et al.: Femoral ... pp. 163-169

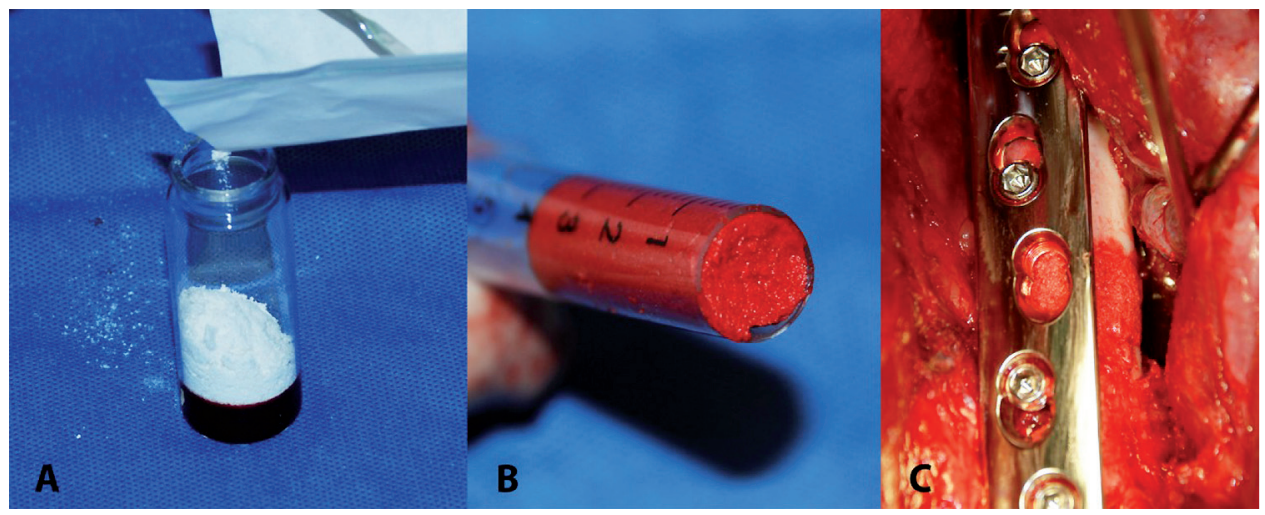

Fig. 1. Experimental group A: A - preparation of the scaffold by mixing autogenous blood with powder substances; B - paste injectable scaffold inside of the syringe before application; C- paste injectable scaffold in the femoral defect after application

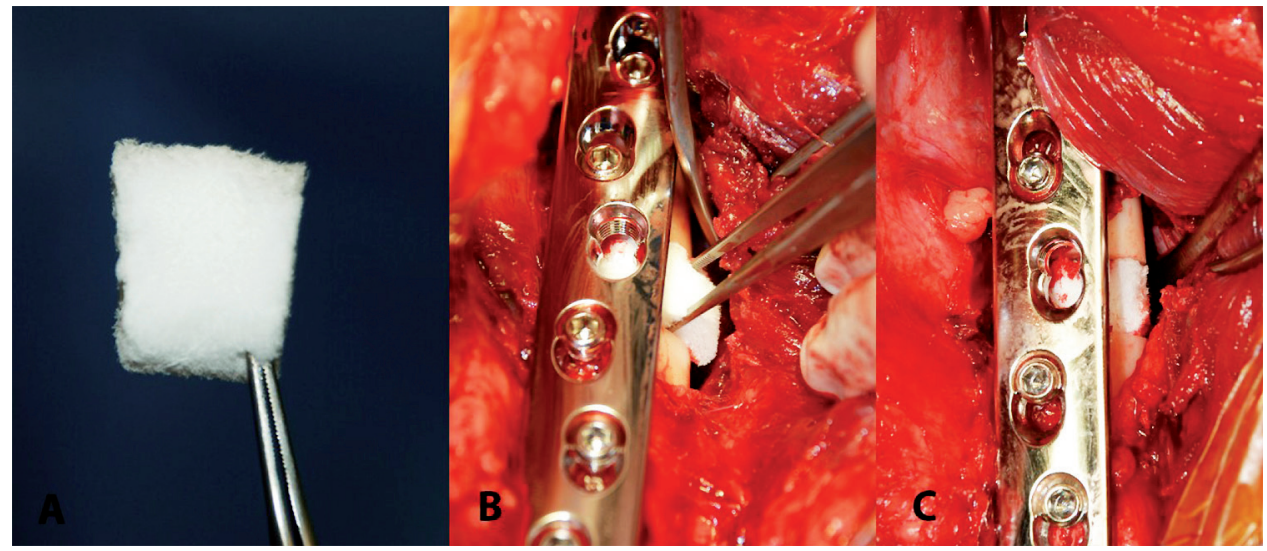

Fig. 2. Experimental group B: A - prefabricated porous 3D cylinder scaffold; B - scaffold application using of the "press and fit" method; C - 3D porous cylindrical scaffold in the femoral defect after application 
Plate II

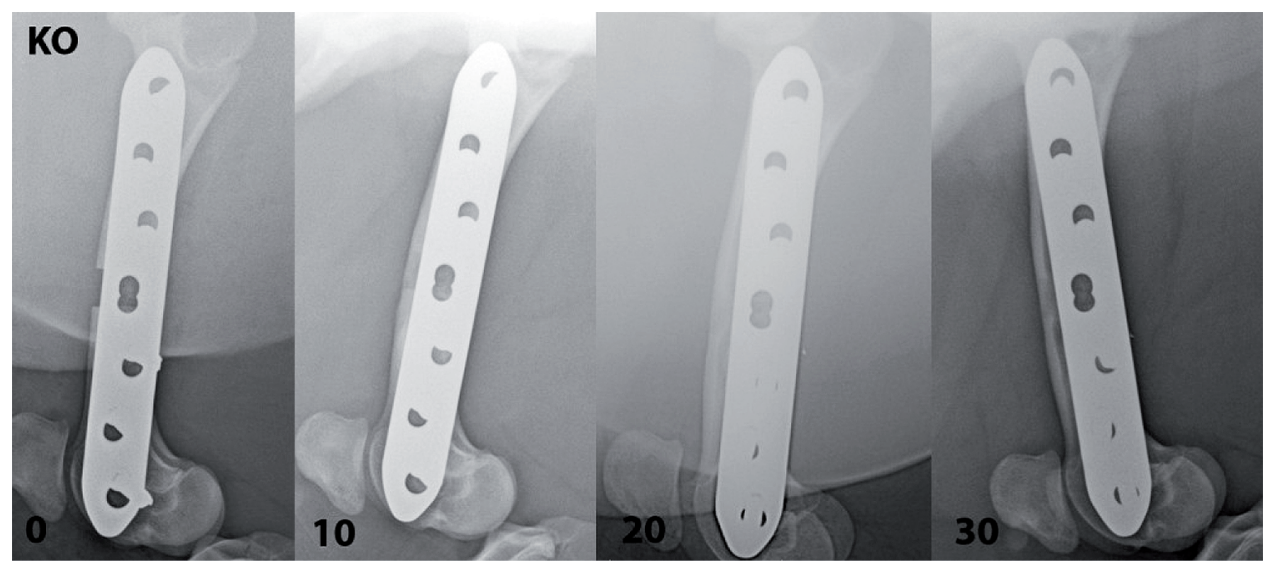

Fig. 3. Radiological evaluation of bone healing: 0, 10, 20 and 30 weeks after implantation - control group

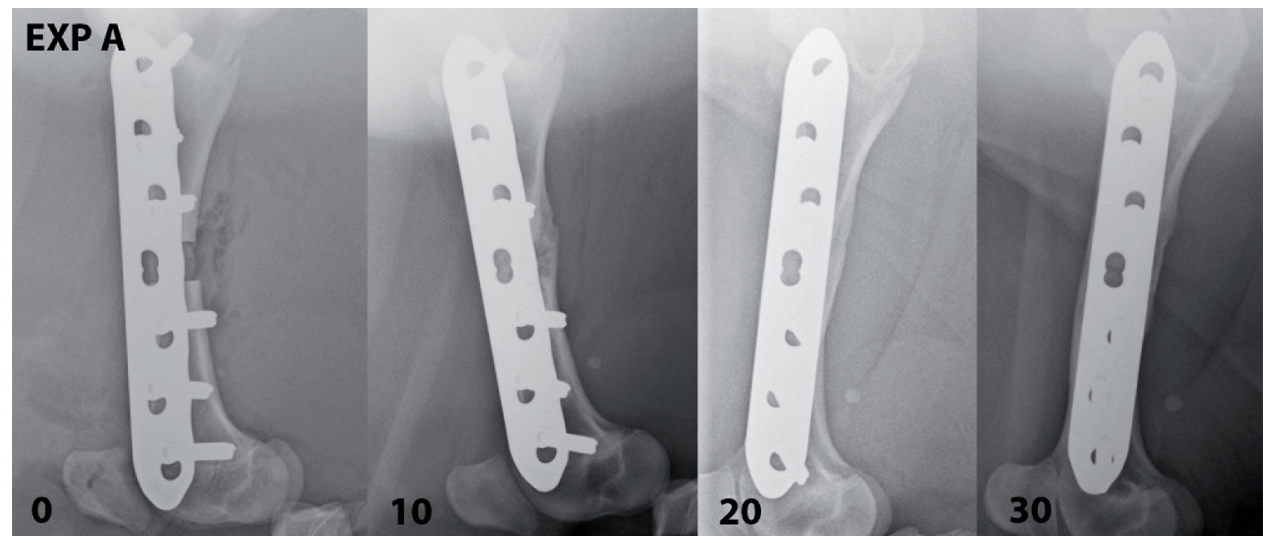

Fig. 4. Radiological evaluation of bone healing: 0, 10, 20 and 30 weeks after implantation - Group EXP A, injectable paste scaffold

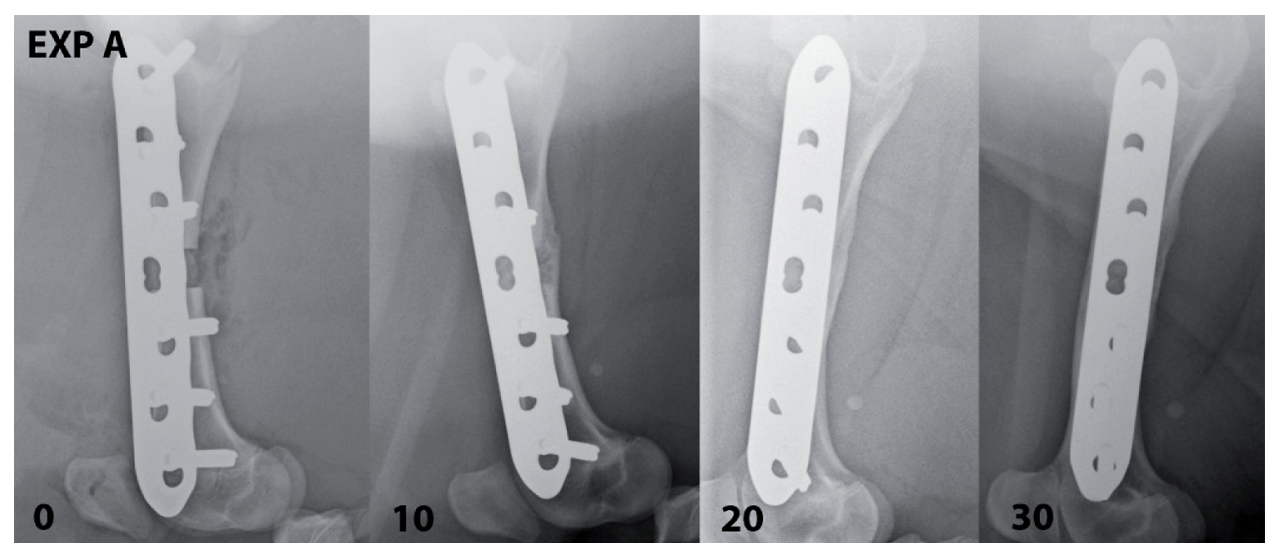

Fig. 5. Radiological evaluation of bone healing: 0, 10, 20 and 30 weeks after implantation - Group EXP B, prefabricated porous 3D cylinder scaffold 